\title{
Integration Model to Support Configuration of Product Architecture and Supply Chain Design
}

\author{
Israel Atakulu ${ }^{1}$, Timofey Shalpegin ${ }^{2}$, David C. Wynn ${ }^{1}$ \\ ${ }^{1}$ University of Auckland, Department of Mechanical Engineering \\ ${ }^{2}$ University of Auckland, Information Systems and Operations Management
}

\begin{abstract}
Supply chain management is a necessary concept for manufacturing firms as it deals with the coordination and integration of interactions of collaborating partners to realize products. The product's architecture is important to incorporating those interactions as it is typically established at the early stages of Product Development and impacts on the costs of interactions (collaboration) within the supply chain. In this paper, a mixed-integer nonlinear model is developed to help minimize the total collaborative costs for organizations involved in collaborative product development. The model concurrently determines the optimal configuration and level of aggregation of modules while minimizing collaborative costs. The application of the model is illustrated using a printer case study. Possible extensions to the approach are highlighted.
\end{abstract}

Keywords: Product Architecture; Collaborative costs; Interface Management; Supply Chain design

\section{Introduction}

In the modern manufacturing industry, increased globalization, rapid improvements in information/logistics technology and growing quality demands from customers have increased the pressure on organizations to stay competitive and meet high demands in the least possible amount of time. To keep up with these demands, organizations with a leading role in new product development (referred to in this paper as Focal Companies) concurrently work with other sub-organizations (suppliers) by allocating them a large and growing proportion of subsystems/modules for development. A recent example of this was the Boeing 787 Dreamliner project with Boeing, the Focal Company, and outsourcing over $70 \%$ of its subsystems to external suppliers.

Considering such issues, research that aims to incorporate Concurrent Engineering with supply chain considerations has emerged. Researchers have referred to this area as $3 \mathrm{D}$ Concurrent Engineering (3D-CE) (Fixson, 2005). Many of those researchers have pointed out the benefits of 3D-CE such as improved supply chain performance, e.g. Ellram, Tate, $\&$ Carter (2007). More recent published papers have been two-dimensional focusing on decisions affecting the product and supply chain domain (e.g. Chiu \& Okudan, 2014; Nepal, Monplaisir, \& Famuyiwa, 2012; Gan \& Grunow, 2016).

However, only limited efforts have been made towards implementing integration models at the early stages of product design to improve collaborative product development processes, in particular most address problems that occur after the physical design phase (i.e. after module aggregation) and focus on supplier selection. This paper contributes to 
the growing product architecture-supply chain design research by proposing an integration model that can help the FC to link the product architecture and supply chain. The model can be applied by the FC to inform decision making towards an optimal trade off of internal and external collaborative costs. The above problems are formulated as a mixed-integer nonlinear programming model (MINLP).

The next subsections briefly describe various necessary concepts. Section 2 provides a review of the relevant literature on concurrent product architecture and supply chain design. In section 3, the proposed model to evaluate product and supply chain decisions is introduced and then formulated as a mathematical decision model. Section 4 provides a case study to demonstrate the proposed model, then presents and discusses the results. Finally, Section 5 concludes the paper with some suggested directions for future work.

\subsection{Product Architecture}

Product architectures specify what subsystems/modules will comprise the final product and their respective functions and connectivity. There are two main types of product architectures highlighted in the literature, indicating the degree to which the product is modular or integral. This paper is mainly focused on the modular type of architecture and considers the situation where some of those modules would be partly or fully realized concurrently as a collaborative effort. The product architecture has an influence on the supply chain configuration by specifying the interfaces between modules to be allocated to suppliers. The representation of these interactions during the product development process can be facilitated through the use of various tools such as the Design Structure Matrix, which is described briefly next.

\subsection{Overview of the Design Structure Matrix (DSM)}

The Design Structure Matrix (DSM) is a commonly-used approach in many disciplines like systems engineering, engineering design/management (e.g., Browning, 2001, Danilovic \& Sandkull, 2005, Danilovic \& Browning, 2007). Steward (1981) introduced the DSM as a project modeling tool that captures the relationships such as components/subsystems in a matrix form thereby outlining the product's architecture. It can be a useful tool to help decision makers to examine a product by providing a clear view of the interdependencies that exists between the products elements. The DSM is applied in three ways in this research - as a decomposition tool to determine a product's architecture and internal interactions, as an integration tool in order to group chunks into modules, and lastly as a way to represent supplier and focal company interactions to enable formulation of an optimization method. For more on the DSM readers are referred to Browning (2001) or Eppinger and Browning (2012).

\section{Literature Review}

The relevant work found in the literature is classified into two categories: frameworks that provide insight into the relevant issues and existing literature, and mathematical modeling 
approaches that propose optimization/simulation tools to aid concurrent product architecture and supply chain design. These two categories are briefly discussed in the next two subsections.

\subsection{Frameworks}

Fixson (2005) proposed a framework that serves as a platform for product design on the product architecture dimension which corresponds to a given operational strategy in the supply chain domain such as resource planning, delivery schedule etc. The framework can help determine the benefits and drawbacks of such operational strategies relative to given product architectures. Pero, Abdelkafi, Sianesi, \& Blecker (2010) developed a conceptual framework that outlines how new product development and supply chain variables are mutually connected to each other and how they can determine a new product performance. They show that aligning product features to supply chain features improves performance while any misalignment may be as a result of an over or under-engineered supply chain. Ülkü \& Schmidt (2011) presented some guidelines for the design of products and supply chain, focusing on the links between product architecture decisions such as the products modularity versus integrality index and the supply chain configuration decisions such as make-buy decisions. Their findings suggested that the choice of product architecture should rely on the market, firm and supply chain structure. Gan \& Grunow (2016) introduced a framework to help trade-off between concurrent product-supply chain attributes. They also illustrated how different product and supply chain system archetypes and their corresponding strategies can be identified. For more information, the reader is referred to Pashaei \& Olhager's (2015) holistic review of the literature on the relationship between product architecture and supply chain design.

\subsection{Mathematical Modeling Approaches}

The majority of models proposed in this category address mainly supplier selection decisions and modularization of the product architecture. Modularization of a product's architecture in particular was the focus of most papers found here - where it is viewed as a strategy that can significantly improve the performance of a new product. For example, Chiu \& Okudan (2014) consider the impact of the modularity level (product architectural decisions) on the supply chain performance (i.e. production lead time, transportation costs and manufacturing processes). Krikke, Bloemhof-Ruwaard, \& Van Wassenhove (2003) developed a quantitative modeling technique to address the modularity of product architecture and the design structure of the supply chain network concurrently, using different parameter settings including alternative product designs, decentralized versus centralized processing etc. Nepal et al. (2012) proposed a multi-objective optimization method to minimize supply chain cost and maximize supply chain compatibility rating, to be able to match the product's architecture strategy to that of the supply chain design during the early stages of product development. Other researchers have considered supply chains on a global level. For instance, ElMaraghy \& Mahmoudi (2009) proposed an integer linear decision support tool to ascertain the optimal location of global supply chain networks. They concurrently studied the currency exchange rate and the optimal product structure at different sites in order to minimize total cost using an industrial example. In this paper, we 
Part II: Product Architecture Design

develop a modeling approach that is unique in the sense that it outlines optimal collaborative scenarios (supplier selection) for the FC while considering the architectural decision of what level of aggregation of modules should be chosen by the Focal Company simultaneously.

\section{The Modelling Method}

A four-stage procedure is proposed which includes acquisition of product and supply chain data, conducting the relevant evaluation, and then optimization.

Step I - Elicit data for the product and supply chain. The Design Structure Matrix is used to represent the product's architecture. The DSM represents the key architectural chunks and how they interact, thereby outlining the basis for coordination relationships between FC and suppliers that would develop the different modules. The set of module configurations to be considered are manually identified. Each module configuration is a possible grouping of the chunks into modules that can be potentially outsourced, and is identified considering the product architecture and the ways that it physically makes sense to group chunks together, considering assembly and other issues. At this step the FC has to also decide the number of suppliers to include in the realization of the product.

Step II - Evaluate and calculate indices for the product and supply chain, for each module configuration. Two key procedures are applied here drawing on the literature e.g., Novak \& Eppinger (2001). Firstly, the more interconnected the modules are internally and externally, the more difficult it is to coordinate development thereby causing higher collaborative efforts. We assume that the effort is a predictor for costs and is quadratic, to be able to efficiently model the interactions. This quadratic model is backed up in the literature by many researchers especially in operations management (e.g. Ülkü \& Schmidt, 2011). Secondly, the greater the number of chunks in a module, the higher the collaborative efforts to develop that particular module since there will be more interactions required to manage as per the product's architecture.

Step III - Compute an optimization model - The different module configurations are considered and the possible supply chain structures for each configuration are optimized.

Step IV - Select the best product architecture-supply chain configuration that deploys best use of resources for the focal company, in terms of minimizing total collaborative cost.

\subsection{Mathematical Formulation}

The model uses the following notation:

$M=$ Modules/subsystems of a product $\left\{1,2, \ldots, N_{m}\right\}$

$S=$ Suppliers assigned to a Module $\left\{1,2, \ldots, \mathrm{N}_{\mathrm{s}}\right\}$

$F=$ Focal Company assigned to a module

The decision variables are as follows:

$X_{m s}=$ Variable indicating supplier $s$ is assigned to module $m$ (Binary variable). 1 indicates that the supplier $s$ has been assigned to a particular module $m$, and 0 - otherwise 
$X_{m f}=$ Variable indicating that the $\mathrm{FC}$ retains Module $m$ (Binary variable).

The parameters are as follows:

$C_{m}=$ Estimated complexity of module $m$. This is modeled as the sum of the internal geometric/physical connections between chunks and the number of chunks in the module, derived from the DSM. It influences the collaborative costs of developing the module as explained in the objective function below.

$U_{i j}=$ Estimated collaborative efforts of FC and suppliers for modules $i$ and $j$ if they are not developed by the same supplier. This is modelled as the sum of interactions between modules and the number of modules in the DSM.

\subsection{Objective Function}

The objective function is the Total Collaborative Cost (TCC) of the Focal Company, which is to be minimized. The cost is modeled as a quadratic function of the effort as earlier mentioned. The TCC can be described as follows: Min \{Internal Collaborative Cost (ICC) + External Collaborative Cost (ECC) $\}$. The Internal Collaborative Cost summarizes the internal cost incurred by the Focal Company when retaining a module $M$ and the supplier's internal cost charged to the FC for realizing modules $M$ assigned by the FC. This is expressed mathematically as:

ICC $=\overrightarrow{X_{f}} \vec{C}+\sum_{j=1}^{N_{s}} \overrightarrow{X_{s}} \vec{C}$

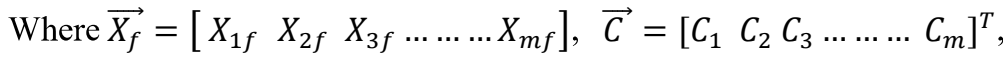

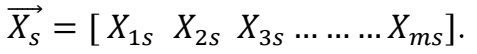

The External Collaborative Cost represents the cost incurred by the Focal Company when coordinating the design of the modules assigned to suppliers externally. This cost includes the cost involved in establishing a well-defined interface between two or more suppliers and the ongoing communication needed to coordinate realization of the design. It is modelled mathematically as:

$E C C=\sum_{i=1}^{N_{m}}\left(X_{i f} \vec{U}_{i}\left(I-\overrightarrow{X_{f}}\right)+\sum_{j=1}^{N_{S}} X_{i j} \vec{U}_{i}\left(I-\overrightarrow{X_{J}}\right)\right)$

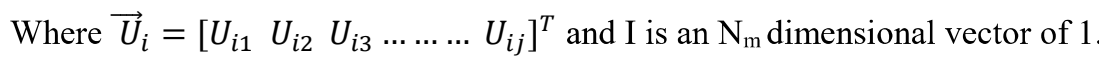


The total collaborative cost of the Focal Company is the sum of (1) and (2) above. $\left(I-\overrightarrow{X_{S}}\right)$ ensures that the collaborative costs is set to zero when the same supplier is assigned modules that share the same communication interface. It is assumed that the cost charged to the Focal Company by the suppliers that have been assigned modules that share the same interfaces is negligible. $\left(I-\overrightarrow{X_{f}}\right)$ ensures zero or negligible costs when the FC retains two modules that share the same communication interface.

\subsection{Constraints}

$\overrightarrow{X_{f}}+\sum_{j=1}^{N_{s}} \overrightarrow{X_{J}}=I$

Where I is an $\mathrm{N}_{\mathrm{m}}$-dimensional vector of 1 . This constraint ensures that the FC or supplier can only be assigned to one module at a time and all modules must be assigned.

\section{Application}

\subsection{Overview and Assumptions}

The model is demonstrated using a reverse engineering case study of a Canon Pixma MG2560 printer (Fig. 1). The printer was selected as it is realistically complex and has a suitable number of possible module configurations to demonstrate the approach. The data required for the model were obtained by disassembling and structural decomposition of the printer. The printer was decomposed based on the physical connectivity between parts, which were grouped as modules where feasible considering the assembly sequence and documented in a Bill of Materials table for easy representation in the DSM. Parts such as screws were excluded and the decomposition was focused at the main physical parts of the printer, which as an incrementally developed product, would be known by the company at the early development stage (once the physical design is established) when the supplier allocation would be considered. The total number of chunks identified after decomposition was 136. The decomposition procedure of the printer was based on some assumptions:

Assumption 1: The product architecture consists of a fully assembled configuration and is assumed to be already established when the method is applied, so that it can be used to determine feasible module configurations that could be used for supplier allocation.

Assumption 2: The product components/chunks can be grouped into subassemblies by considering the likely assembly sequence. The chunks that make up a sub-assembly are referred to as a module.

Assumption 3: The supply chain considered here consists of a focal company and its tier 1 suppliers. Lower tier suppliers are not explicitly modelled here for simplicity. For the case study, number of suppliers also is also kept to a maximum of 3 . 


\section{Atakulu, T. Shalpegin, D. C. Wynn}

\subsection{Procedure}

The four-stage procedure was carried out for the printer. After the disassembly, DSMs were used to represent the printer's architecture, each showing a different realistic module configuration for possible supplier allocations. Then, the necessary indices were calculated. The different module configurations were input together with the supply chain data and solved as a MINLP. At first, the decision variables were set to default with the FC retaining all the modules (value of 1 for all values of $\mathrm{X}_{\mathrm{mf}}$ ) and suppliers having no modules (value of 0 for $\mathrm{X}_{\mathrm{ms}}$ ). This is because the $\mathrm{FC}$, which is the principal organization, initially has all the modules and decides how much/what to allocate to suppliers. The optimal solutions were found by triggering different starting points for the optimization procedure to ensure the true optimal solution was found. The solution was run until no change occurred to the optimal solution.

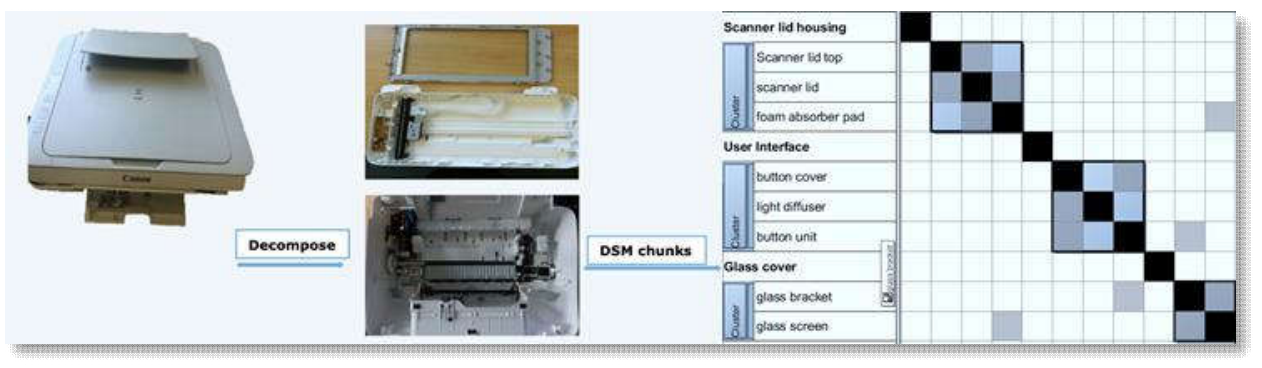

Figure 1. Decomposition Procedure

\subsection{Results and implications}

As can be seen in Figure 2, the results from this case suggests that increasing the number of modules included in the final product (by reducing the number of parts/chunks per module) affects the total costs faced by the Focal Company. When an emphasis is placed by the Focal Company on aggregating fewer modules to reduce their external collaboration costs, this adversely affects the total collaborative costs because the FC would have to use up a lot of its resources to manage its internal collaboration.

Some general implications can be suggested. Having fewer modules decreases the need for external collaboration, but increases the internal collaborative costs significantly and has an undesirable effect on the total collaborative costs of the FC. Similarly, the suppliers that are allocated modules have a greater burden of coordinating their personnel internally and in turn might charge the FC more. On the other hand, increasing the number of modules in the product architecture reduces the internal collaborative costs for the FC because more modules are being assigned to suppliers, but increases the external collaboration costs as the $\mathrm{FC}$ would have to dedicate more attention to manage the communication interfaces with/between suppliers.

This is evident in Figure 2 which shows how increasing the number of modules from the lowest possible value leads to a decrease in total collaborative cost of the FC, because the FC has more options to leverage the suppliers by allocating the modules in different ways. 


\section{Part II: Product Architecture Design}

However, at some point, increasing the number of modules to assign to suppliers becomes a burden to the FC as it has to use up a lot of resources coordinating among the suppliers. There is an optimal point for the $\mathrm{FC}$ - at which the external and internal collaborative costs present an optimal trade-off that minimizes total collaboration costs.

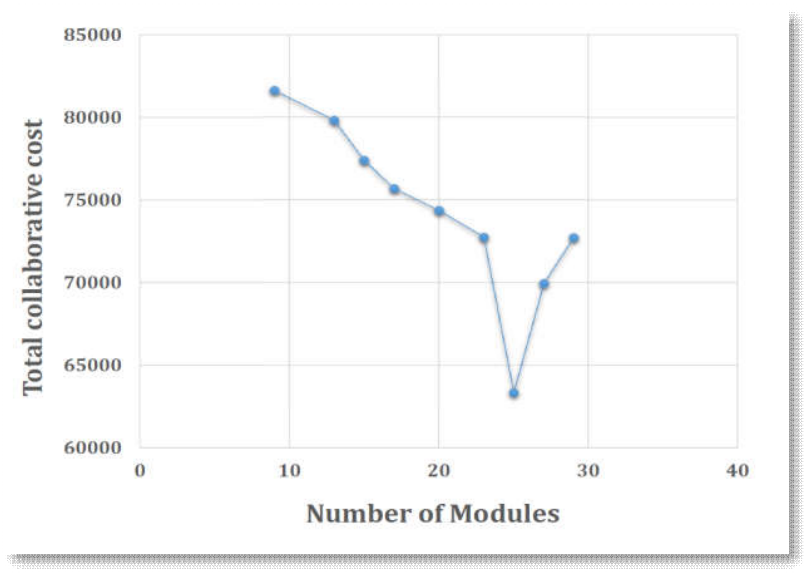

Figure 2. Optimal internal-external collaborative costs scenario that minimizes total collaborative costs.

In this case, optimal collaborative costs can be achieved when the product architecture is aggregated into 25 modules. Put another way, in this case the FC will benefit from decomposing the product as far as 25 modules, after which a diminishing return sets in.

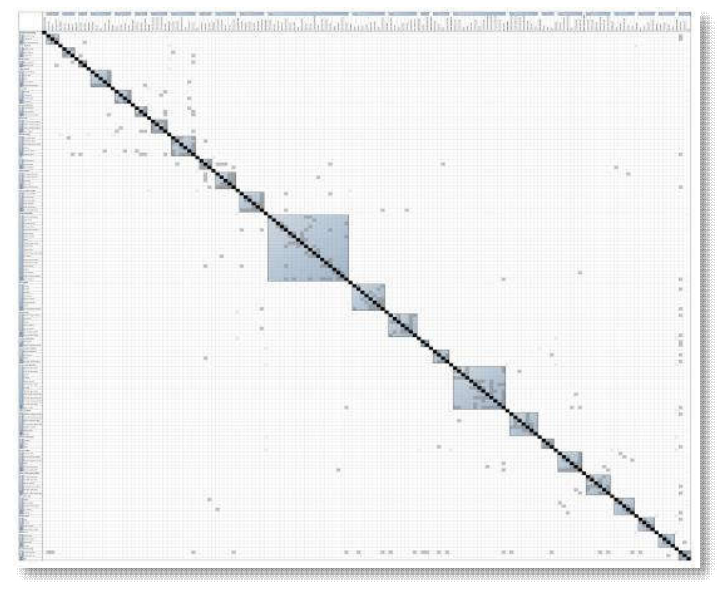

Figure 3. DSM showing optimal level of decomposition of modules

The result shows that there is an optimal level of aggregation (level of decomposition) of modules that minimizes the total collaboration costs, and the FC can use this to maximize 


\section{Atakulu, T. Shalpegin, D. C. Wynn}

strategic objectives. This point is where the internal and external collaboration costs are optimal. The example thereby shows how the model can help to investigate the effectiveness of product architecture decomposition strategies and supplier allocation strategies concurrently. It can be applied by FCs to determine the right level of modules/level of aggregation of modules to suit the company's objective in the creation of a new product and supply chain configuration. It can also be used to outline the optimal level of internal and external collaborative costs strategy adopted by the FC.

\section{Conclusion}

An integrative model was proposed that considers aspects of product and supply chain design to concurrently determine the optimal configuration and level of aggregation of modules that also minimizes collaborative costs for the FC. The model was illustrated using a printer case study. It serves as a support tool for FCs to make decisions such as module configuration, the optimal level of module aggregation, and also to identify the optimal internal and external costing scenario when collaborating with suppliers.

Some limitations are apparent. The external collaborative costs were all assumed to carry equal weights in the DSM but in reality the costs incurred would be on a case-by-case basis. The possible clusterings into modules were also identified manually (although realistically based on a physical product decomposition). While every effort was made to ensure only realistic module configurations were considered in the printer analysis, it is possible that other combinations exist. The result obtained using this model has only been applied to the printer case study described and needs to be tested in an industrial context. Finally, the method focuses only on collaborative costs. In practice, many additional issues impact on module aggregation and supplier allocation decisions, including management of product families and platforms, strategies for standardization vs innovation, and functionality of modules. Such issues would need to be considered as well as the optimal module aggregation with respect to collaborative cost.

Several issues warrant further research in addition to addressing the limitations above. Firstly, more decision variables and parameters can incorporated and formulated as optimization problems. Particularly, parameters such as the capacities and expertise level of the suppliers can have an important implication on the configuration of modules in the product's architecture and the collaborative costs of the FC. Incorporating such parameters would enable sensitivity analyses to be carried out and more specific managerial findings to be drawn. Finally, the parameters used here are all deterministic values; however, in a new product development effort, some degree of uncertainty is expected especially at the early design stages. Incorporating uncertainty will therefore be considered in future work.

\section{References}

Browning, T. R. (2001). Applying the design structure matrix to system decomposition and integration problems: a review and new directions. IEEE Transactions on Engineering Management, 48(3), 292-306. 
Part II: Product Architecture Design

Chiu, M.-C., \& Okudan, G. (2014). An investigation on the impact of product modularity level on supply chain performance metrics: an industrial case study. Journal of Intelligent Manufacturing, 25(1), 129-145.

Danilovic, M., \& Browning, T. R. (2007). Managing complex product development projects with design structure matrices and domain mapping matrices. International journal of project management, 25(3), 300-314.

Danilovic, M., \& Sandkull, B. (2005). The use of dependence structure matrix and domain mapping matrix in managing uncertainty in multiple project situations. International journal of project management, 23(3), 193-203.

Ellram, L. M., Tate, W. L., \& Carter, C. R. (2007). Product-process-supply chain: an integrative approach to three-dimensional concurrent engineering. International Journal of Physical Distribution \& Logistics Management, 37(4), 305-330.

ElMaraghy, H. A., \& Mahmoudi, N. (2009). Concurrent design of product modules structure and global supply chain configurations. International Journal of Computer Integrated Manufacturing, 22(6), 483-493.

Eppinger, S. D., \& Browning, T. R. (2012). Design structure matrix methods and applications. MIT press.

Fixson, S. K. (2005). Product architecture assessment: a tool to link product, process, and supply chain design decisions. Journal of Operations Management, 23(3), $345-$ 369.

Gan, T.-S., \& Grunow, M. (2016). Concurrent product and supply chain design: a literature review, an exploratory research framework and a process for modularity design. International Journal of Computer Integrated Manufacturing, 29(12), 1255-1271.

Krikke, H., Bloemhof-Ruwaard, J., \& Van Wassenhove, L. (2003). Concurrent product and closed-loop supply chain design with an application to refrigerators. International Journal of Production Research, 41(16), 3689-3719.

Nepal, B., Monplaisir, L., \& Famuyiwa, O. (2012). Matching product architecture with supply chain design. European Journal of Operational Research, 216(2), 312325.

Novak, S., \& Eppinger, S. D. (2001). Sourcing by design: Product complexity and the supply chain. Management science, 47(1), 189-204.

Pashaei, S., \& Olhager, J. (2015). Product architecture and supply chain design: a systematic review and research agenda. Supply Chain Management: An International Journal, 20(1), 98-112.

Pero, M., Abdelkafi, N., Sianesi, A., \& Blecker, T. (2010). A framework for the alignment of new product development and supply chains. Supply Chain Management: An International Journal, 15(2), 115-128.

Steward, D. V. (1981). The design structure system: A method for managing the design of complex systems. IEEE Transactions on Engineering Management(3), 71-74.

Ülkü, S., \& Schmidt, G. M. (2011). Matching product architecture and supply chain configuration. Production and Operations Management, 20(1), 16-31.

Contact: I.N. Atakulu, University of Auckland, Department of Mechanical Engineering, 7

Symonds Street, 1010, Auckland, New Zealand, +64221829081, iata582@aucklanduni.ac.nz. 